Pacific Journal of Mathematics

REPRESENTATION OF VECTOR-VALUED FUNCTIONS BY 


\title{
REPRESENTATION OF VECTOR-VALUED FUNCTIONS BY LAPLACE TRANSFORMS
}

\author{
W. RICKER
}

In this note various criteria are given which solve the following problem. Given a locally convex space $X$ and an $X$-valued function on $(0, \infty)$, when does there exist an $X$-valued function on $[0, \infty)$, usually required to have certain specific properties such as continuity, integrability, etc., whose Laplace transform is the given function? Some of these criteria are new even in Banach spaces.

1. Introduction. Many problems in classical analysis are subsumed under the theory of semigroups of linear operators. This is particularly true of Cauchy's problem which arises in the theory of partial differential equations. An important problem is the generation of semigroups, that is, to determine which operators are the infinitesimal generator of a semigroup. For strongly continuous semigroups a successful approach to this problem can be based on the Post-Widder inversion formula for Laplace transforms, which provides a connection between the semigroup and the resolvent map of its infinitesimal generator [12]. However, as W. Feller repeatedly emphasized (see for example [7]-[10]), the theory of strongly continuous semigroups is often inadequate in practice. For example, it is not applicable to the theory of diffusion processes and problems arising from applications to stochastic processes. The semigroup arising from the heat equation may also fail to be strongly continuous in certain function spaces; see $\S 3$ of [2]. It seems plausible that a theory for semigroups which are not necessarily strongly continuous could still be based on the method of Laplace transforms. However, the representation theorems available for Laplace transforms of vector-valued functions appear to be too restrictive for such applications.

Accordingly, the aim of this note is to provide more general criteria which guarantee that a given vector-valued function is a Laplace transform. The results are based on the classical Widder inversion operators (see §2). The functions involved will assume their values in locally convex spaces. This is not because they are more general than Banach spaces, but because they provide the natural setting for problems of this type.

A novelty of the note occurs in $\$ 5$ where sufficient conditions are presented which ensure that a given function is the Laplace transform of a 
Pettis integrable function. This is possible due to some recent work of S. Okada which characterizes the completion of the space of strongly measurable, Pettis integrable functions for the topology of convergence in mean, as a space of Pettis integrable functions with values in an auxiliary space containing a copy of the original space. Even for Banach spaces such a criterion was not previously available.

The author would like to thank Professors I. Kluvánek and S. Okada for valuable discussions, especially concerning $§ 5$.

2. Notations and preliminaries. For a vector-valued function there are many possible ways of defining measurability and integrability. Some of these definitions may be considered as natural extensions of the numerical-valued case. This is in particular true for Bochner integrable functions [3]. However, in practice many functions fail to have such strong properties and hence, more general definitions are needed. In this section we give the basic definitions and results concerning measurability and integrability of vector-valued functions which are needed in the sequel.

Let $X$ be a locally convex Hausdorff space, always assumed to be quasi-complete. The space of continuous linear functionals on $X$ and the space of all linear functionals on $X$ are denoted by $X^{\prime}$ and $X^{*}$, respectively.

An $X$-valued vector measure is a $\sigma$-additive map $m: \mathscr{M} \rightarrow X$, whose domain $\mathscr{M}$ is a $\sigma$-algebra of subsets of some non-empty set $\Omega$. For each $x^{\prime} \in X^{\prime}$, the complex-valued measure $E \mapsto\left\langle m(E), x^{\prime}\right\rangle, E \in \mathscr{M}$, is denoted by $\left\langle m, x^{\prime}\right\rangle$. Its variation is denoted by $\left\langle m, x^{\prime}\right\rangle \mid$.

For each continuous seminorm $q$ on $X$, let $U_{q}^{\circ}$ denote the polar of the neighbourhood, $q^{-1}([0,1])$, of zero. Then the $q$-semivariation of a vector measure $m: \mathscr{M} \rightarrow X$ is the set function $q(m)$ defined by

$$
q(m)(E)=\sup \left\{\left|\left\langle m, x^{\prime}\right\rangle\right|(E) ; x^{\prime} \in U_{q}^{\circ}\right\}, \quad E \in \mathscr{M} .
$$

For each continuous seminorm $q$ on $X$, the function $m \mapsto q(m)(\Omega)$ is a seminorm for the space of $X$-valued vector measures on $\mathscr{M}$. The so-defined topology for the space of $X$-valued vector measures on $\mathscr{M}$ is called the topology of convergence in mean.

Let $m: \mathscr{M} \rightarrow X$ be a vector measure. A complex-valued, $\mathscr{M}$-measurable function $f$ on $\Omega$ is said to be $m$-integrable if it is integrable with respect to every measure $\left\langle m, x^{\prime}\right\rangle, x^{\prime} \in X^{\prime}$, and if, for every set $E \in \mathscr{M}$, there exists an element $\int_{E} f d m$ of $X$ such that

$$
\left\langle\int_{E} f d m, x^{\prime}\right\rangle=\int_{E} f d\left\langle m, x^{\prime}\right\rangle
$$


for each $x^{\prime} \in X^{\prime}$. The element $\int_{E} f d m$ is denoted simply by $(f m)(E)$. The $X$-valued map

$$
f m: E \mapsto(f m)(E), \quad E \in \mathscr{M},
$$

is called the indefinite integral of the function $f$ with respect to the measure $m$. The Orlicz-Pettis lemma implies that it is a vector measure.

Let $\mathscr{M}$ be a $\sigma$-algebra of subsets of a non-empty set $\Omega$ and $\lambda$ be a countably additive measure on $\mathscr{M}$, either complex-valued or non-negative extended real-valued.

A function $f: \Omega \rightarrow X$ is said to be Pettis integrable with respect to $\lambda$ or briefly, $\lambda$-integrable, if the function $\left\langle f, x^{\prime}\right\rangle: w \mapsto\left\langle f(w), x^{\prime}\right\rangle, w \in \Omega$, is $\lambda$-integrable for each $x^{\prime} \in X^{\prime}$, and if, for very set $E \in \mathscr{M}$, there exists an element $\int_{E} f d \lambda$ of $X$ such that

$$
\left\langle\int_{E} f d \lambda, x^{\prime}\right\rangle=\int_{E}\left\langle f, x^{\prime}\right\rangle d \lambda
$$

for each $x^{\prime} \in X^{\prime}$. The element $\int_{E} f d \lambda$ is denoted simply by $(f \lambda)(E)$. The Orlicz-Pettis lemma implies that the indefinite integral of $f$, that is, the map $f \lambda: E \mapsto(f \lambda)(E), E \in \mathscr{M}$, is an $X$-valued vector measure. The element $(f \lambda)(\Omega)$ is denoted simply by $\lambda(f)$.

Let $f$ be an $X$-valued function on $\Omega$. If the function $\left\langle f, x^{\prime}\right\rangle$ is $\mathscr{M}$-measurable for each $x^{\prime} \in X^{\prime}$, then $f$ is said to be scalarly measurable. If there exists a sequence $f_{n}: \Omega \rightarrow X, n=1,2, \ldots$, of $\mathscr{M}$-simple functions based on sets of finite $\lambda$-measure such that $f(w)=\lim _{n \rightarrow \infty} f_{n}(w)$, for $\lambda=$ a.e. point $w \in \Omega$, then $f$ is called strongly measurable.

A topological space is called a Suslin space if it is the continuous image of a complete, separable metric space. The properties of Suslin spaces are systematically exposed in [20]; see also [21]. For such spaces there is a strong relationship between the various notions of measurability.

LEMMA 2.1. Let $X$ be a Suslin, locally convex space and $\lambda: \mathscr{M} \rightarrow[0, \infty]$ be a $\sigma$-finite measure. The following conditions for a function $f: \Omega \rightarrow X$ are equivalent.

(i) $f$ is Borel measurable, that is, $f^{-1}(B) \in \mathscr{M}$ for every Borel subset $B$ of $X$.

(ii) $f$ is scalarly measurable.

(iii) $f$ is strongly measurable.

Proof. See [21; Theorem 1] and [13; IV Theorem 2.3].

The following results are concerned with the representation of weakly compact operators defined on $L^{1}$-spaces. 
LEMMA 2.2. Let $\lambda: \mathscr{M} \rightarrow[0, \infty]$ be a $\sigma$-finite measure and $f: \Omega \rightarrow X$ be a scalarly measurable function with relatively weakly compact range. Then $f$ is Pettis integrable with respect to each measure $\psi \lambda, \psi \in L^{1}(\lambda)$, and the linear operator $\Phi: L^{1}(\lambda) \rightarrow X$ given by

$$
\Phi(\psi)=(\psi \lambda)(f), \quad \psi \in L^{1}(\lambda),
$$

is weakly compact.

Proof. The integrability of $f$ with respect to each finite measure $\psi \lambda$, $\psi \in L^{1}(\lambda)$, follows from [21; Lemma A]. A simple bipolar argument shows that if $B$ denotes the closed balanced convex hull of the range of $f$, then

$$
\left|\left\langle\int_{E} f \psi d \lambda, x^{\prime}\right\rangle\right| \leq\|\psi\|_{1}, \quad x^{\prime} \in B^{\circ},
$$

for each $\psi \in L^{1}(\lambda)$. Hence, $\left\{\Phi(\psi) ; \psi \in \mathrm{L}^{1}(\lambda),\|\psi\|_{1} \leq 1\right\}$ is a subset of $B$. It follows from the Krein-Smulian theorem for quasi-complete spaces that $B$ is weakly compact and hence, that $\Phi$ is a weakly compact operator.

It is rather the converse of Lemma 2.2 which is needed in the sequel. This is the Dunford-Pettis theorem [4, VI Theorem 8.10], which in the case of a separable Banach space $X$, states that any weakly compact operator $\Phi: L^{1}(\lambda) \rightarrow X$ is of the form (1). In fact, any function $f$ representing $\Phi$ in the form (1) is necessarily strongly measurable with $\lambda$-a.e. value in a weakly compact set. Accordingly, the integral in (1) is in the sense of Bochner. As noted in [21], the Dunford-Pettis theorem can be extended to a larger class of spaces.

Proposition 2.3. Let $X$ be a Suslin, locally convex space and $\lambda$ : $\mathscr{M} \rightarrow[0, \infty]$ be a $\sigma$-finite measure. If $\Phi: L^{1}(\lambda) \rightarrow X$ is a weakly compact operator, then there exists a ( $\lambda$-unique) strongly measurable function $f$ : $\Omega \rightarrow X$ with values in a weakly compact set such that $\Phi$ is given by (1).

Proof. Let $K$ denote the weak closure of $\left\{\Phi(\psi) ; \psi \in L^{1}(\lambda),\|\psi\|_{1} \leq 1\right\}$. Since $X$ is Suslin for the weak topology $[21 ;$ p. 69] and $K$ is weakly closed, it follows that $K$ is Suslin and compact for the weak topology and hence, that $K$ is metrizable for the weak topology [1; Ch. 9, Corollarie 2, Appendix 1]. Accordingly, $K$ has a countable base for the weak topology. The result follows from [4; VI Theorem 8.2] and Lemma 2.1.

If the function representing $\Phi$ in (1) is not required to be strongly measurable, then the following extension of the Dunford-Pettis theorem is 
possible. The proof, which uses the theory of liftings, was suggested by Dr. B. Jefferies.

Proposition 2.4. Let $\lambda: \mathscr{M} \rightarrow[0, \infty]$ be a complete, $\sigma$-finite measure and $X$ be a quasi-complete, locally convex space. If $\Phi: L^{1}(\lambda) \rightarrow X$ is a weakly compact operator, then there exists a weakly Borel measurable function $f: \Omega \rightarrow X$ with relatively weakly compact range such that $\Phi$ is given by (1).

Proof. Let $\Omega_{k}, k=1,2, \ldots$, be disjoint measurable sets, each of finite $\lambda$-measure, whose union is $\Omega$. If $K$ denotes the closure of the convex set $\left\{\Phi(\psi) ; \psi \in \mathrm{L}^{1}(\lambda),\|\psi\|_{1} \leq 1\right\}$, then $K$ is weakly compact.

For each $k=1,2, \ldots$, define a set function $m_{k}: \mathscr{M} \cap \Omega_{k} \rightarrow X$ by

$$
m_{k}(E)=\Phi\left(\chi_{E}\right), \quad E \in \mathscr{M} \cap \Omega_{k} .
$$

Then $m_{k}$ is a vector measure whose $\lambda$-average range, $\left\{m_{k}(A) / \lambda(A) ; A \in\right.$ $\left.\mathscr{M} \cap \Omega_{k}, \lambda(A)>0\right\}$, is contained in $K$. Hence, each measure $m_{k}, k=$ $1,2, \ldots$, has relatively weakly compact range contained in $K$. It follows (see [5; Theorem 1.2] or [22; Corollary 1.7]) that there is a weakly Borel measurable function $f_{k}: \Omega_{k} \rightarrow K$ such that

$$
m_{k}(E)=\left(f_{k} \lambda\right)(E), \quad E \in \mathscr{M} \cap \Omega_{k},
$$

for each $k=1,2, \ldots$

Let $f=\sum_{k=1}^{\infty} f_{k}$. Then $f$ is a weakly Borel measurable function on $\Omega$ with range contained in $K$. If $E \in \mathscr{M}$ has finite $\lambda$-measure, then it is easily shown that

$$
\Phi\left(\chi_{E}\right)=(f \lambda)(E)
$$

A standard argument using (2), the weak continuity of $\Phi$ and the density of the $\mathscr{M}$-simple functions in $L^{1}(\lambda)$, shows that $\Phi$ is given by (1).

It is worth noting that slightly more has been shown than asserted in the Proposition. Namely, it follows from [5; Theorem 1.2] that the image measure $\lambda \circ f^{-1}$ is actually (weakly) Radon. Hence, using an argument similar to that in the proof of IV Corollary 1.2 of [14], it follows that if $g$ : $\Omega \rightarrow X$ is another weakly Borel measurable function representing $\Phi$ such that $\lambda \circ g^{-1}$ is (weakly) Radon, then $f$ and $g$ agree $\lambda$-a.e.

For the remainder of this section $\Omega=[0, \infty), \mathscr{M}$ is the $\sigma$-algebra of Borel sets in $\Omega$ and $\lambda$ is Lebesgue measure.

A function $f:(0, \infty) \rightarrow X$ is said to be a Laplace transform if there 
exists a scalarly measurable function $\phi: \Omega \rightarrow X$ which is Pettis integrable with respect to the measure

$$
\lambda_{t}: E \mapsto \int_{E} e^{-w t} d w, \quad E \in \mathscr{M}
$$

for each $t>0$, and such that

$$
f(t)=\lambda_{t}(\phi)=\int_{0}^{\infty} e^{-w t} \phi(w) d w, \quad t>0 .
$$

The relation (4) is denoted by $f=\hat{\phi}$.

Let $f$ be an infinitely differentiable, complex-valued function defined on $(0, \infty)$. The Widder differential operators $L_{k}, k=1,2, \ldots$, are defined by

$$
L_{k}(f)(w)=(-1)^{k}(k !)^{-1}(k / w)^{k+1} f^{(k)}(k / w), \quad w>0 .
$$

The following result follows from [24; VII Theorem 11b].

LEMMA 2.5. Let $f$ be a complex-valued function with derivatives of all orders in $(0, \infty)$. If for each $k=1,2, \ldots$,

$$
\int_{0}^{v} L_{k}(f) d w=O(v), \quad v \rightarrow \infty
$$

then $f(\infty)$ exists, and

$$
\lim _{k \rightarrow \infty} \int_{0}^{\infty} e^{-t w} L_{k}(f)(w) d w=f(t)-f(\infty), \quad t>0
$$

If a function $f:(0, \infty) \rightarrow X$ has weak derivatives of all orders, in the sense of Definition 3.2.3 in [12], then the Widder differential operators (5) can be applied to $f$ giving a sequence of $X$-valued functions

$$
w \mapsto L_{k}(f)(w), \quad w>0,
$$

for $k=1,2, \ldots$

3. Laplace transforms of continuous functions. In Chapter VII, $\$ 5$ of [24] it is shown that if $\phi$ is a continuous, complex-valued function on $[0, \infty)$ with a limit at infinity, then

$$
\lim _{k \rightarrow \infty} L_{k}(\hat{\phi})(w)=\phi(w)
$$

uniformly in $0 \leq \mathrm{w}<\infty$. Several authors have noted various analogoues of (6) in the case when $\phi$ is a vector-valued function; see for example [12; Theorem 6.3.5] and [16; Theorem 2.5] for Banach spaces or [6; Auxiliary 
Lemma 3.2] for more general spaces. This section is concerned rather with the converse problem, namely when a given function is the Laplace transform of some continuous function.

Let $X$ be a quasi-complete, locally convex space. The linear space of all continuous functions $\phi:[0, \infty) \rightarrow X$ for which $\lim _{w \rightarrow \infty} \phi(w)$ exists in $X$, is denoted by $\mathscr{C}_{l}(X)$. For each continuous seminorm $q$ on $X$, define a seminorm $\tilde{q}$ on $\mathscr{C}_{l}(X)$ by

$$
\tilde{q}(\phi)=\sup \{q(\phi(w)): w \geq 0\}, \quad \phi \in \mathscr{C}_{l}(X) .
$$

Then $\mathscr{C}_{1}(X)$ is a sequentially complete, locally convex space.

Let $\phi \in \mathscr{C}_{l}(X)$. Since $\phi$ is Lusin $\lambda_{t}$-measurable, $t>0[15 ; \S 2]$, and bounded it follows that $\phi$ is Pettis integrable with respect to each of the finite, regular measures $\lambda_{t}, t>0$, given by (3); see for example [15; Lemma 4] extended to quasi-complete spaces. Hence, the Laplace transform of $\phi$ is defined.

Let $\mathscr{F}(X)$ denote the linear space of all $X$-valued functions $f$ on $(0, \infty)$, with weak derivatives of all orders (see $\S 2)$, such that

$$
L_{k}(f)(0)=\lim _{w \rightarrow 0+} L_{k}(f)(w),
$$

exists in $X$ for each $k=1,2, \ldots$ It is tacitly assumed that whenever $f \in \mathscr{F}(X)$, each of the maps $L_{k}(f):(0, \infty) \rightarrow X, k=1,2, \ldots$, has been extended to $[0, \infty)$ by declaring its value at zero to be the limit $(8)$.

LEMMA 3.1. Let $\phi \in \mathscr{C}_{l}(X)$. Then for each $k=1,2, \ldots$,

$$
\lim _{w \rightarrow 0+} L_{k}(\hat{\phi})(w)=\phi(0) \text {. }
$$

In particular, $\hat{\phi}$ belongs to $\mathscr{F}(X)$.

Proof. It follows from (4) and (5) that $\hat{\phi}$ has weak derivatives of all orders (equal to (17) for each $k=1,2, \ldots$ ) and that

$$
L_{k}(\hat{\phi})(w)=(k !)(k / w)^{k+1} \int_{0}^{\infty} s^{k} e^{-k s / w} \phi(s) d s, \quad w>0,
$$

for each $k=1,2, \ldots$. Substituting $u=k s / w$ gives

$$
L_{k}(\hat{\phi})(w)=(k !)^{-1} \int_{0}^{\infty} u^{k} e^{-u} \phi(u w / k) d u, \quad w>0
$$

for each $k=1,2, \ldots$

Fix $k$. Let $w_{n} \rightarrow 0+$. Define bounded, continuous functions $\phi_{n}$ : $[0, \infty) \rightarrow \mathrm{X}, n=1,2, \ldots$, by

$$
\phi_{n}(u)=(k !)^{-1} u^{k} e^{-u / 2} \phi\left(u w_{n} / k\right), \quad u \geq 0 .
$$


If $\psi(u)=(k !)^{-1} u^{k} e^{-u / 2} \phi(0), u \geq 0$, then $\phi_{n} \rightarrow \psi$ in $\mathscr{C}_{l}(X)$ as $n \rightarrow \infty$. Hence, it follows from the inequalities

$q\left(\int_{0}^{\infty} \phi_{n} d \lambda_{1 / 2}-\int_{0}^{\infty} \psi d \lambda_{1 / 2}\right) \leq \int_{0}^{\infty} q\left(\phi_{n}-\psi\right) d \lambda_{1 / 2}, \quad n=1,2, \ldots$,

valid for each continuous seminorm $q$ on $X$, and the Dominated Convergence Theorem for the finite measure $\lambda_{1 / 2}$ that

$$
\begin{aligned}
\lim _{n \rightarrow \infty} L_{k}(\hat{\phi})\left(w_{n}\right) & =\lim _{n \rightarrow \infty} \int_{0}^{\infty} \phi_{n}(u) d \lambda_{1 / 2}(u) \\
& =\int_{0}^{\infty} \psi(u) d \lambda_{1 / 2}(u)=\phi(0) .
\end{aligned}
$$

Proposition 3.2. A function $f:(0, \infty) \rightarrow X$ is the Laplace transform of a function in $\mathscr{C}_{l}(X)$, if and only if,

(i) $f \in \mathscr{F}(X)$

(ii) $f$ vanishes at infinity with respect to the weak topology on $X$,

(iii) for each $k=1,2, \ldots$, and $x^{\prime} \in X^{\prime}$,

$$
\int_{0}^{v} L_{k}\left(\left\langle f, x^{\prime}\right\rangle\right)(w) d w=O(v), \quad v \rightarrow \infty,
$$

(iv) the sequence $\left\{L_{k}(f)\right\}_{k=1}^{\infty}$ is Cauchy in $\mathscr{C}_{l}(X)$.

If such a function exists in $\mathscr{C}_{l}(X)$, then it is unique.

Proof. If (i) - (iv) hold, then there exists a function $\phi \in \mathscr{C}_{l}(X)$ such that $L_{k}(f) \rightarrow \phi$, uniformly on $[0, \infty)$. In particular, $L_{k}\left(\left\langle f, x^{\prime}\right\rangle\right) \rightarrow$ $\left\langle\phi, x^{\prime}\right\rangle$, uniformly on $[0, \infty)$, for each $x^{\prime} \in X^{\prime}$. Accordingly, if $\varepsilon>0$ and $t>0$, then there exists an integer $k_{0}=k_{0}\left(\varepsilon, t, x^{\prime}\right)$ such that $k>k_{0}$ implies

$$
\left|L_{k}\left(\left\langle f, x^{\prime}\right\rangle\right)(w)-\left\langle\phi(w), x^{\prime}\right\rangle\right|<\varepsilon t, \quad w \geq 0 .
$$

Thus, for $k>k_{0}$,

$$
\begin{gathered}
\left|\int_{0}^{\infty} e^{-w t} L_{k}\left(\left\langle f, x^{\prime}\right\rangle\right)(w) d w-\int_{0}^{\infty} e^{-w t}\left\langle\phi(w), x^{\prime}\right\rangle d w\right| \\
<\varepsilon t \int_{0}^{\infty} e^{-w t} d w=\varepsilon
\end{gathered}
$$

It follows from Lemma 2.5 that

$$
\left\langle f(t), x^{\prime}\right\rangle=\int_{0}^{\infty} e^{-w t}\left\langle\phi(w), x^{\prime}\right\rangle d w, \quad t>0 .
$$

Since this holds for all $x^{\prime} \in X^{\prime}$, it follows that the function $f$ is the Laplace transform of $\phi$. 
Conversely, suppose that $f=\hat{\phi}$ for some $\phi \in \mathscr{C}_{l}(X)$. Lemma 3.1 implies that $f \in \mathscr{F}(X)$ which is (i). If $x^{\prime} \in X^{\prime}$, then $M_{x^{\prime}}=$ $\sup \left\{\left|\left\langle\phi(w), x^{\prime}\right\rangle\right| ; w \geq 0\right\}$ is finite and

$$
\left|\left\langle f(t), x^{\prime}\right\rangle\right| \leq M_{x^{\prime}} \int_{0}^{\infty} e^{-w t} d w=t^{-1} M_{x^{\prime}} \rightarrow 0, \quad t \rightarrow \infty .
$$

Hence, (ii) holds.

It follows from (9) that for each $x^{\prime} \in X^{\prime}$ and $k=1,2, \ldots$,

$L_{k}\left(\left\langle f, x^{\prime}\right\rangle\right)(w)=(k !)^{-1}(k / w)^{k+1} \int_{0}^{\infty} s^{k} e^{-k s / w}\left\langle\phi(s), x^{\prime}\right\rangle d s, \quad w>0$.

Since for each $w>0$ and $k=1,2, \ldots$,

$$
(k !)^{-1}(k / w)^{k+1} \int_{0}^{\infty} s^{k} e^{-k s / w} d s=1,
$$

it follows that

$$
\left|\int_{0}^{v} L_{k}\left(\left\langle f, x^{\prime}\right\rangle\right)(w) d w\right| \leq M_{x^{\prime}} \int_{0}^{v} d w=O(v), \quad v \rightarrow \infty,
$$

which is (iii).

The proof of (iv) is analogous to that of [24; VII Theorem 5b]; see for example [23; Theorem 1.12]. It is for this part of the argument that the existence of $\lim _{w \rightarrow \infty} \phi(w)$ is needed. Hence, the conditions (i) - (iv) are necessary.

Denote by $\mathscr{C}_{b}(X)$ the linear space of all bounded, continuous $X$-valued functions on $[0, \infty)$. If $\mathscr{C}_{b}(X)$ is equipped with the topology induced by the seminorms (7), for each continuous seminorms (7), for each continuous seminorm $q$ on $X$, then it is a sequentially complete, locally convex space.

An examination of the proof of Proposition 3.2 shows that the sufficiency of the conditions (i)-(iv) did not require the existence of $\lim _{w \rightarrow \infty} \phi(w)$. Accordingly, if $f:(0, \infty) \rightarrow X$ is a function satisfying the conditions (i)-(iii) of Proposition 3.2 and such that the sequence $\left\{L_{k}(f)\right\}_{k=1}^{\infty}$ is Cauchy in $\mathscr{C}_{b}(X)$, then $f$ is the Laplace transform of a unique function in $\mathscr{C}_{b}(X)$.

The space $\mathscr{C}_{b}(X)$ cannot be replaced by the space of all continuous $X$-valued functions on $[0, \infty)$ as the Laplace transform of such functions may not be defined.

4. Laplace transforms of bounded measurable functions. The proofs of those criteria which guarantee that a given Banach space valued function is the Laplace transform of a bounded, strongly measurable 
function and which are formulated in terms of a family of inversion operators, are usually based on the Dunford-Pettis theorem; see for example [17], [19], [23], [25]. As noted in §2, versions of the Dunford-Pettis theorem can be extended to a larger class of spaces. In this section the problem of representing a given function as the Laplace transform of a bounded measurable function is considered in a more general setting.

LEMMA 4.1. Let $f:(0, \infty) \rightarrow X$ be a function having weak derivatives of all orders. Then there exists a closed, separable subspace $Y$ of $X$ which contains the range of $L_{k}(f)$ for each $k=1,2, \ldots$. If $Y$ is Suslin for the relative topology induced by $X$, then the functions $L_{k}(f), k=1,2, \ldots$, are strongly measurable. In particular, if $X$ is a Fréchet space, then each function $L_{k}(f), k=1,2, \ldots$, is strongly measurable.

Proof. Since $L_{k}(f)$ is weakly continuous for each $k=1,2, \ldots$, it follows that $R=\left\{L_{k}(f)(w) ; \mathrm{w}>0, \mathrm{k}=1,2, \ldots\right\}$ is weakly separable. Accordingly, the smallest closed linear subspace $Y$ of $X$ containing $R$ is weakly separable, hence separable. If $Y$ is Suslin, then Lemma 2.1 implies the functions $L_{k}(f), k=1,2, \ldots$, are strongly measurable. In particular, this is the case if $X$ is a Fréchet space as separable Fréchet spaces are Suslin.

Let $X$ be a quasi-complete, locally convex space. Let $f:(0, \infty) \rightarrow X$ be a function having weak derivatives of all orders. Being weakly continuous, each of the functions $L_{k}(f), k=1,2, \ldots$, is weakly (Lusin) $\psi \lambda$-measurable $[15 ; \S 2]$ with respect to each of the finite, regular measures $\psi \lambda$, $\psi \in L^{1}(\lambda)$, where $\lambda$ denotes Lebesgue measure on $[0, \infty)$. If each function $L_{k}(f), k=1,2, \ldots$, is Pettis integrable with respect to each of the measures $\psi \lambda, \psi \in L^{1}(\lambda)$, then a sequence of linear operators $\Phi_{k}(f)$ : $L^{1}(\lambda) \rightarrow X, k=1,2, \ldots$, can be defined by

$$
\Phi_{k}(f)(\psi)=\int_{0}^{\infty} L_{k}(f)(w) \psi(w) d w, \quad \psi \in L^{1}(\lambda) .
$$

D. V. Widder characterized those functions on $(0, \infty)$ which are the Laplace transforms of bounded measurable functions [24; VII, \$16]. Using the theory of Bochner integration this result was extended to Banach space valued functions in [17; Theorem 4] and [25]. The following two propositions can be interpreted as a natural extension of this sequence of results.

Proposition 4.2. Let $X$ be a Fréchet space and $f:(0, \infty) \rightarrow X a$ function. Then $f$ is the Laplace transform of an $X$-valued, strongly measurable function on $[0, \infty)$ with relatively weakly compact range, if and only if, 
(i) f has weak derivatives of all orders.

(ii) $f$ vanishes at infinity with respect to the weak topology on $X$,

(iii) for each $k=1,2, \ldots$, the function $L_{k}(f)$ is Pettis integrable with respect to each measure $\psi \lambda, \psi \in L^{1}(\lambda)$, and

(iv) the set $\left\{\Phi_{k}(f)(\psi) ; \psi \in L^{1}(\lambda),\|\psi\|_{1} \leq 1, k=1,2,\right\}$ is relatively weakly compact.

Proof. Let $K$ denote the weak closure of $\left\{\Phi_{k}(f)(\psi) ; \psi \in L^{1}(\lambda),\|\psi\|_{1}\right.$ $\leq 1, k=1,2, \ldots\}$. It follows from [11; (1), p. 39] that any sequence in $K$ has a weakly convergent subsequence. Using this fact it follows from an argument as in the proof of Theorem 9.1 of [19], that there is an increasing sequence of integers $\left\{k_{i}\right\}_{i=1}^{\infty}$ such that the limits

$$
\text { (weak) } \lim _{i \rightarrow \infty} \Phi_{k_{l}}(f)(\psi), \quad \psi \in L^{1}(\lambda),
$$

exist in $X$. Hence, the linear operator $\Phi(f): L^{1}(\lambda) \rightarrow X$ whose value at each point $\psi \in L^{1}(\lambda)$ is declared to be the limit (13), is weakly compact.

Lemma 4.1 implies that there exists a closed, separable subspace $Y$ of $X$ which contains the set $\left\{L_{k}(f)(w) ; w>0, k=1,2, \ldots\right\}$. If $\psi \in L^{1}(\lambda)$ is finitely-valued, then it is clear from (12) that $\Phi_{k}(f)(\psi) \in Y$, for each $k=1,2, \ldots$, and hence, that $\Phi_{k}(f)(\psi) \in Y$ for each $\psi \in L^{1}(\lambda)$ and $k=1,2, \ldots$. Then (13) shows that $\Phi(f)$ assumes its values in $Y$. By Proposition 2.3 there is a strongly measurable, $Y$-valued function $\phi$ defined on $[0, \infty)$ with values in a weakly compact subset of $Y$, hence of $X$, such that

$$
\Phi(f)(\psi)=\int_{0}^{\infty} \phi(w) \psi(w) d w, \quad \psi \in L^{1}(\lambda) .
$$

Since $w \mapsto e^{-w t}, w \geq 0$, belongs to $L^{1}(\lambda)$ for each $t>0$, it follows from (13) that

$$
\int_{0}^{\infty} e^{-w t} \phi(w) d w=\text { (weak) } \lim _{i \rightarrow \infty} \int_{0}^{\infty} e^{-w t} L_{k_{\imath}}(f) d w
$$

for each $t>0$. The boundedness of $K$ implies that for each $x^{\prime} \in X^{\prime}$ there is a positive constant $M_{x^{\prime}}$ satisfying

$$
\sup _{\|\psi\|_{1} \leq 1}\left|\int_{0}^{\infty} \psi(w) L_{k}\left(\left\langle f, x^{\prime}\right\rangle\right)(w) d w\right|=\sup _{\|\psi\|_{1} \leq 1}\left|\left\langle\Phi_{k}(f)(\psi), x^{\prime}\right\rangle\right| \leq M_{x^{\prime}}
$$

for $k=1,2, \ldots$. That is,

$$
\underset{w \geq 0}{\operatorname{ess-sup}}\left|L_{k}\left(\left\langle f, x^{\prime}\right\rangle\right)(w)\right| \leq M_{x^{\prime}}, \quad k=1,2, \ldots
$$


Also condition (ii) implies that $\lim _{t \rightarrow \infty}\left\langle f(t), x^{\prime}\right\rangle=0$, for each $x^{\prime} \in X^{\prime}$. Hence, Lemma 2.5 and (15) imply that for each $x^{\prime} \in X^{\prime}$,

$$
\left\langle f(t), x^{\prime}\right\rangle=\left\langle\int_{0}^{\infty} e^{-w t} \phi(w) d w, x^{\prime}\right\rangle, \quad t>0 .
$$

That is, $f=\hat{\phi}$.

Conversely, suppose that $\phi:[0, \infty) \rightarrow X$ is strongly measurable and has relatively weakly compact range. Let $K$ denote the closed balanced convex hull of the range of $\phi$. Since for each $k=1,2, \ldots$, the function $w \mapsto w^{k} e^{-w t}, w \geq 0$, is $\lambda$-integrable for each $t>0$, it follows from Lemma 2.2 that

$$
(-1)^{k} \int_{0}^{\infty} w^{k} e^{-w t} \phi(w) d w
$$

is an element of $X$, for each $k=1,2, \ldots$, and $t>0$. It is then easily verified that $\hat{\phi}$ has weak derivatives of all orders; in fact $(\hat{\phi})^{(k)}(t)$ is equal to (17) for each $k=1,2, \ldots$, and $t>0$.

If $x^{\prime} \in X^{\prime}$, then there is a constant $M_{x^{\prime}}>0$ such that $\left|\left\langle\phi(w), x^{\prime}\right\rangle\right| \leq$ $M_{x^{\prime}}$, for each $w \geq 0$. Accordingly, the estimate

$$
\left|\left\langle\hat{\phi}(t), x^{\prime}\right\rangle\right| \leq \int_{0}^{\infty} e^{-w t}\left|\left\langle\phi(w), x^{\prime}\right\rangle\right| d w \leq M_{x^{\prime}} t^{-1}, \quad t>0,
$$

shows that $\hat{\phi}$ vanishes at infinity with respect to the weak topology on $X$.

It follows from (5) and (17) that $L_{k}(\hat{\phi})(w)$ is given by (9) for each $k=1,2, \ldots$, and $w>0$. Since $\int_{0}^{\infty} \psi(w) \phi(w) d w \in K$ for each $\psi \in L^{1}(\lambda)$ with $\|\psi\|_{1} \leq 1$ (see proof of Lemma 2.2) it follows from (9) and (11) that $L_{k}(\hat{\phi})(w) \in K$ for each $k=1,2, \ldots$, and $w>0$. Lemma 2.2 then implies that condition (iii) is satisfied.

It is clear from (12) that $\Phi_{k}(\hat{\phi})(\psi) \in K$ whenever $\psi$ is a finitely-valued integrable function with $\|\psi\|_{1} \leq 1$ and hence, it follows that $\Phi_{k}(\hat{\phi})(\psi)$ $\in K$ for all $k=1,2, \ldots$, and $\psi \in L^{1}(\lambda)$ such that $\|\psi\|_{1} \leq 1$ (recall that $K$ is closed, balanced and convex). Hence, condition (iv) is satisfied.

The above proof is based on the fact that if a subset of a metrizable space is weakly compact then it is also weakly sequentially compact. Suslin spaces also have this property. This follows from a theorem of Hausdorff which asserts the equivalence of compactness and sequential compactness in metric spaces and the fact that a weakly compact subset of a Suslin space is metrizable (see the proof of Proposition 2.3). Accordingly, Proposition 4.2 is valid if in its statement the space $X$ is any Suslin, locally convex space.

D. H. Fremlin introduced a class of topological spaces called angelic spaces which have the property that a subset is compact, if and only if, it 
is sequentially compact. As already noted, many locally convex spaces are angelic for the weak topology. A systematic exposition of such spaces can be found in [11; Chapter 3].

Proposition 4.3. Let $X$ be a quasi-complete, locally convex space which is angelic for the weak topology. A function $f:(0, \infty) \rightarrow X$ is the Laplace transform of an $X$-valued, weakly Borel measurable function on $[0, \infty)$ with relatively weakly compact range, if and only if, if satisfies the conditions (i)-(iv) of Proposition 4.2.

Proof. Suppose that $f$ satisfies the hypotheses of Proposition 4.2. Using the fact that $X$ is angelic for the weak topology, it follows as in the proof of Proposition 4.2 that there is an increasing sequence of positive integers $\left\{k_{i}\right\}_{l=1}^{\infty}$ and a weakly compact operator $\Phi(f): L^{1}(\lambda) \rightarrow X$ whose value at each point $\psi \in L^{1}(\lambda)$ is given by the limit (13). Proposition 2.4 gives the existence of a weakly Borel measurable function $\phi:[0, \infty) \rightarrow X$ with values in a weakly compact subset of $X$ such that $\Phi(f)$ is given by (14). The proof can now be completed as in the proof of Proposition 4.2.

5. Laplace transforms of integrable functions. Let $\lambda$ denote Lebesgue measure on $[0, \infty)$. It is well known that a complex-valued function $f$ on $(0, \infty)$ is the Laplace transform of an integrable function, if and only if, $f$ has derivatives of all orders, vanishes at infinity and $\left\{L_{k}(f)\right\}_{k=1}^{\infty}$ is a Cauchy sequence in $L^{1}(\lambda)$, [24, VII, Theorem 17a]. If $X$ is a Banach space and $B_{1}(X)$ the space of $X$-valued, Bochner integrable functions defined on $[0, \infty)$, equipped with the usual norm

$$
\|g\|=\int_{0}^{\infty}\|g(w)\| d \lambda(w), \quad g \in B_{1}(X),
$$

then the same criterion characterizes those functions $f:(0, \infty) \rightarrow X$ which are the Laplace transform of members of $B_{1}(X)$, [17, Theorem 5]. The completeness of $B_{1}(X)$ guarantees the existence of a limit, $\phi$, of the sequence $\left\{L_{k}(f)\right\}_{k=1}^{\infty}$, and it follows that $f=\hat{\phi}$. However, as noted previously, the requirement of Bochner integrability is unduely restrictive in practice.

Let $\mathscr{P}_{1}(X)$ denote the space of $X$-valued, Pettis integrable functions defined on $[0, \infty)$, equipped with the topology of convergence in mean of indefinite integrals. If $f:(0, \infty) \rightarrow X$ is a function for which $\left\{L_{k}(f)\right\}_{k=1}^{\infty}$ is a Cauchy sequence in the space $\mathscr{P}_{1}(X)$, then it is not in general possible to deduce the existence of a limit of $\left\{L_{k}(f)\right\}_{k=1}^{\infty}$ in $\mathscr{P}_{1}(X)$. This is due to the 
fact that $\mathscr{P}_{1}(X)$ is not complete. However, if the function whose transform is $f$ need not assume its values in $X$, then this difficulty can be overcome.

Throughout this section $X$ denotes a Fréchet space with topology specified by a sequence of seminorms $q_{n}, n=1,2, \ldots$

Let $Y$ be a locally convex Hausdorff space such that there exists a continuous linear injection of $X$ into $Y$. Then the space $Y^{\prime}$ can be identified with a subspace of $X^{\prime}$ which separates the points of $X$.

A $Y$-valued function $f$ defined on $[0, \infty)$ is said to be $(X, Y)$-Archimedes integrable with respect to $\lambda,[18]$, if there exist vectors $c_{i} \in X$ and Borel sets $E_{i} \subset[0, \infty), i=1,2, \ldots$, of finite $\lambda$-measurable such that

(i) the sequence of sets $\left\{c_{i} \lambda(F) ; F \text { Borel, } F \subseteq E_{i}\right\}_{i=1}^{\infty}$ is summable in $X$, in the sense of [18], and

(ii) if $y^{\prime} \in Y^{\prime}$, then the equality

$$
\left\langle f(w), y^{\prime}\right\rangle=\sum_{i=1}^{\infty}\left\langle c_{i}, y^{\prime}\right\rangle \chi_{E_{i}}(w),
$$

holds for every $w \geq 0$ such that $\sum_{i=1}^{\infty}\left\langle c_{i}, y^{\prime}\right\rangle \mid \chi_{E_{i}}(w)$ is finite.

The indefinite integral of $f$ with respect to $\lambda$ is the $X$-valued vector measure $f \lambda$ given by

$$
(f \lambda)(E)=\sum_{i=1}^{\infty} \lambda\left(E \cap E_{i}\right) c_{i}
$$

for each Borel set $E \subseteq[0, \infty)$.

The vector space of all $(X, Y)$-Archimedes integrable functions on $[0, \infty)$ is denoted by $L_{0}(\lambda ; X, Y)$. Each seminorm $q_{n}, n=1,2, \ldots$, on $X$ induces a seminorm on $L_{0}(\lambda ; X, Y)$ given by the formula

$$
f \mapsto q_{n}(f \lambda)([0, \infty)), \quad f \in L_{0}(\lambda ; X, Y) .
$$

The so defined semi-metric space $L_{0}(\lambda ; X, Y)$ may not be Hausdorff. This can be overcome in the usual way by declaring two elements $f$ and $g$ of $L_{0}(\lambda ; X, Y)$ to be equal if

$$
q_{n}((f-g) \lambda)([0, \infty))=0, \quad n=1,2, \ldots
$$

This is equivalent to the requirement that $\left\langle f, y^{\prime}\right\rangle=\left\langle g, y^{\prime}\right\rangle \lambda$-a.e. for each $y^{\prime} \in Y^{\prime}$. The resulting Hausdorff, locally convex metric space (of equivalence classes) is denoted by $L(\lambda ; X, Y)$. It contains the $X$-valued simple functions as a dense subspace. In particular, if $L_{0}(\lambda ; X, Y)$ is complete, then $L(\lambda: X, Y)$ is a Fréchet space. It is the completion of the space of $X$-valued simple functions on $[0, \infty)$ for the topology of convergence in mean of indefinite integrals.

The existence of spaces $Y$ for which $L_{0}(\lambda ; X, Y)$ is complete is guaranteed by the following result, [18]. 
Proposition 5.1. The locally convex space $L_{0}\left(\lambda ; X, X^{*}\right)$ is complete and contains as dense subspaces the space of $X$-valued simple functions based on sets of finite $\lambda$-measure and the space of strongly measurable, $X$-valued Pettis integrable functions on $[0, \infty)$.

The following result provides sufficient conditions for an $X$-valued function on $(0, \infty)$ to be the Laplace transform of an $X^{\prime *}$-valued, Archimedes integrable function.

Proposition 5.2. Let $f$ be an $X$-valued function having weak derivatives of all orders in $(0, \infty)$ such that $f$ vanishes at infinity with respect to the weak topology on $X$ and $\left\{L_{k}(f)\right\}_{k=1}^{\infty}$ is a Cauchy sequence in $\mathscr{P}_{1}(X)$. Then there exists a unique $\left(X, X^{*}\right)$-Archimedes integrable function on $[0, \infty)$ whose Laplace transform is $f$.

Proof. It follows from Lemma 4.1 that each function $L_{k}(f), k=$ $1,2, \ldots$, is strongly measurable and hence, is $\left(X, X^{\prime *}\right)$-Archimedes integrable. Proposition 5.1 implies that there exists an $\left(X, X^{*}\right)$-Archimedes integrable function $\phi$ such that $L_{k}(f) \rightarrow \phi$ in $L\left(\lambda ; X, X^{*}\right)$. Since the function $w \mapsto e^{-w t}, w \geq 0$, is bounded and measurable for each $t>0$, it follows that $e^{-(\cdot) t} \phi(\cdot)$ is $\left(X, X^{*}\right)$-Archimedes integrable [18; Corollary 4]. Hence, the Laplace transform of $\phi$ is defined and takes its values in $X$.

If $x^{\prime} \in\left(X^{\prime *}\right)^{\prime}$, then also $x^{\prime} \in X^{\prime}$. Accordingly,

$$
\left\langle\int_{0}^{\infty} e^{-w t} \phi(w) d w, x^{\prime}\right\rangle=\int_{0}^{\infty} e^{-w t}\left\langle\phi(w), x^{\prime}\right\rangle d w, \quad t>0 .
$$

Since $\left\langle L_{k}(f), x^{\prime}\right\rangle \rightarrow\left\langle\phi, x^{\prime}\right\rangle$ in $L^{1}(\lambda)$, it follows that (10) is valid for all $x^{\prime} \in\left(X^{*}\right)^{\prime}$, [24; VII Theorem 17a]. Then (18) implies that (16) holds and hence, that $f=\hat{\phi}$.

Let $\psi$ be another $\left(X, X^{\prime *}\right)$-Archimedes integrable function such that $f=\hat{\psi}$. It follows from (18) that for each $x^{\prime} \in\left(X^{\prime *}\right)^{\prime}$,

$$
\int_{0}^{\infty} e^{-w t}\left\langle\phi(w), x^{\prime}\right\rangle d w=\int_{0}^{\infty} e^{-w t}\left\langle\psi(w), x^{\prime}\right\rangle d w, \quad t>0 .
$$

Hence, $\left\langle\phi, x^{\prime}\right\rangle=\left\langle\psi, x^{\prime}\right\rangle \lambda$-a.e. [4; VIII Lemma 1.15] for each $x^{\prime} \in\left(X^{\prime *}\right)^{\prime}$, that is, $\phi=\psi$ in the space $L\left(\lambda ; X, X^{\prime *}\right)$.

It is worth noting that for particular spaces $X$ it may be possible to replace the space $X^{\prime *}$ in Proposition 5.2 by a substantially smaller space. For example, if $X$ is a separable Hilbert space and $\Gamma$ is a complete orthonormal basis for $X^{\prime}$, then the vector space $\mathbf{C}^{\Gamma}$, consisting of all complex-valued functions on $\Gamma$ equipped with the natural linear operations, is a Fréchet space with respect to the topology of pointwise convergence. Furthermore, $X$ is continuously imbedded in $\mathbf{C}^{\Gamma}$ and the 
space $L\left(\lambda ; X, \mathbf{C}^{\Gamma}\right)$ is complete, $[18 ; \S 2]$. Accordingly, if a function $f$ : $(0, \infty) \rightarrow X$ satisfies the hypotheses of Proposition 5.2, then there exists a unique $\left(X, \mathbf{C}^{\Gamma}\right)$-Archimedes integrable function on $[0, \infty)$ whose Laplace transform is $f$.

The formulation of the converse statement to Proposition 5.2 does not require the completeness of the space $L(\lambda ; X, Y)$.

Proposition 5.3. Let $Y$ be a locally convex space in which $X$ is continuously included. If $\phi$ is an $(X, Y)$-Archimedes integrable function on $[0, \infty)$, then

(i) $\hat{\phi}$ has weak derivatives of all orders and vanishes at infinity with respect to the weak topology on $X$,

(ii) the $X$-valued functions $L_{k}(\hat{\phi}), k=1,2, \ldots$, are strongly measurable and Pettis $\lambda$-integrable, and

(iii) the sequence $\left\{L_{k}(\hat{\phi})\right\}_{k=1}^{\infty}$ is Cauchy in the space $\mathscr{P}_{1}(X)$.

Proof. Let $m=\phi \lambda$ denote the indefinite integral of $\phi$ with respect to $\lambda$. Then $m$ is an $X$-valued vector measure whose Laplace transform is $\hat{\phi}$, that is,

$$
\hat{\phi}(t)=\int_{0}^{\infty} e^{-w t} \phi(w) d w=\int_{0}^{\infty} e^{-w t} d m(w), \quad t>0 .
$$

It follows that $\hat{\phi}$ has weak derivatives of all orders and that $L_{k}(\hat{\phi})$ is Pettis integrable for each $k=1,2, \ldots$, [23; Theorem 1.6]. The strong measurability of the functions $L_{k}(\hat{\phi}), k=1,2, \ldots$, follows from Lemma 4.1.

Let $t_{n} \rightarrow \infty$. Then the sequence of functions $w \mapsto e^{-w t_{n}}, w \geq 0$, for each $n=1,2, \ldots$, converges pointwise to $\chi_{\{0\}}$. It follows from the Dominated Convergence Theorem for vector measures that

$$
\lim _{n \rightarrow \infty}\left\langle\hat{\phi}\left(t_{n}\right), x^{\prime}\right\rangle=\left\langle m(\{0\},), x^{\prime}\right\rangle,
$$

for each $x^{\prime} \in X^{\prime}$. That is, $\lim _{t \rightarrow \infty} \hat{\phi}(t)=m(\{0\})$ weakly in $X$. However, if $y^{\prime} \in Y^{\prime}$, then $\left\langle\phi, y^{\prime}\right\rangle \in L^{1}(\lambda)$ and

$$
\left\langle m(\{0\}), y^{\prime}\right\rangle=\int_{0}^{\infty} \chi_{\{0\}}(w)\left\langle\phi(w), y^{\prime}\right\rangle d w=0 .
$$

Since $Y^{\prime}$ separates points of $X$ it follows that $m(\{0\})=0$ and hence, that $\lim _{t \rightarrow \infty} \hat{\phi}(t)=0$ weakly in $X$.

To verify (iii) let $\varepsilon>0$. Fix a seminorm $q_{n}$ determining the topology of $X$. There exist points $c_{i} \in X, 1 \leq i \leq r$, and Borel sets $E_{i} \subset[0, \infty)$, $1 \leq i \leq r$, of finite Lebesgue measure such that the function $h=\sum_{l=1}^{r} c_{i} \chi_{E_{i}}$ satisfies

$$
q_{n}(h \lambda-\phi h)([0, \infty))<\varepsilon .
$$


Let $\mu=h \lambda-\phi \lambda$. If $k$ is a positive integer, then it follows from Fubini's theorem and the identity (11) that

$$
\begin{aligned}
\int_{0}^{\infty}\left|\left\langle L_{k}(\hat{\phi})(\xi)-L_{k}(\hat{h})(\xi), x^{\prime}\right\rangle\right| d \xi \\
\quad \leq \int_{0}^{\infty}(k !)^{-1}(k / \xi)^{k+1}\left(\int_{0}^{\infty} w^{k} e^{-k w / \xi} d\left|\left\langle\mu, x^{\prime}\right\rangle\right|(w)\right) d \xi \\
\quad=\left|\left\langle\mu, x^{\prime}\right\rangle\right|([0, \infty)),
\end{aligned}
$$

for each $x^{\prime} \in X^{\prime}$. Accordingly,

$$
\begin{aligned}
& q_{n}\left(L_{k}(\hat{\phi}) \lambda-L_{k}(\hat{h}) \lambda\right)([0, \infty)) \\
& \quad \leq \sup \left\{\left|\left\langle\mu, x^{\prime}\right\rangle\right|([0, \infty)) ; x^{\prime} \in U_{q_{n}}^{\circ}\right\} \\
& \quad=q_{n}(h \lambda-\phi \lambda)([0, \infty)) \leq \varepsilon,
\end{aligned}
$$

for each $k=1,2, \ldots$

Choose $\alpha>0$ such that $q_{n}\left(c_{i}\right) \leq \alpha, 1 \leq i \leq r$. Since

$$
\begin{aligned}
& q_{n}\left(L_{k}(\hat{h}) \lambda-L_{l}(\hat{h}) \lambda\right)([0, \infty)) \\
& \quad \leq \sup _{x^{\prime} \in U_{q_{n}}^{\circ}} \sum_{i=1}^{r}\left|\left\langle c_{i}, x^{\prime}\right\rangle\right|\left\|L_{k}\left(\hat{\chi}_{E_{t}}\right)-L_{l}\left(\hat{\chi}_{E_{i}}\right)\right\|_{1},
\end{aligned}
$$

for each $k, l=1,2, \ldots$, and $\chi_{E_{i}} \in L^{1}(\lambda), 1 \leq i \leq r$, there is a positive integer $N$ such that

$$
q_{n}\left(L_{k}(\hat{h}) \lambda-L_{l}(\hat{h}) \lambda\right)([0, \infty)) \leq \varepsilon(\alpha r)^{-1} \sup _{x^{\prime} \in U_{q_{n}}^{\circ}} \sum_{i=1}^{r}\left|\left\langle c_{i}, x^{\prime}\right\rangle\right| \leq \varepsilon,
$$

for all $k, l \geq N$. It follows from (19) and (20) that

$$
q_{n}\left(L_{k}(\hat{\phi}) \lambda-L_{l}(\hat{\phi}) \lambda\right)([0, \infty)) \leq 3 \varepsilon, \quad k, l \geq N .
$$

This shows that the sequence $\left\{L_{k}(\hat{\phi})\right\}_{k=1}^{\infty}$ is Cauchy in $\mathscr{P}_{1}(X)$.

\section{REFERENCES}

[1] N. Bourbaki, Topologie Générale II, Chapitres 5 à 10, Herman, Paris, 1974.

[2] R. Carmona, Regularity properties of Schrödinger and Dirichlet semi-groups, J. Funct. Anal., 33 (1979), 259-296.

[3] J. Diestel and J. J. Uhl, Jr., Vector Measures, Math. Surveys No. 15, Amer. Math. Soc., Providence., R.I., 1977.

[4] N. Dunford and J. Schwartz, Linear Operators I, Interscience Publishers, New York, 1966.

[5] G. A. Edgar, On the Radon-Nikodym property and martingale convergence, Lecture Notes in Mathematics No. 645, Vector Space Measures and Applications II, Proc. of Dublin Conference, Springer-Verlag, 1977. 
[6] H. O. Fattorini, Ordinary differential equations in linear topological spaces II, J. Differential Equations, 6 (1969), 50-70.

[7] W. Feller, The parabolic differential equations and the associated semi-groups of transformations, Ann. of Math., 55 (1952), 468-519.

[8] - Semi-groups of transformations in general weak topologies, Ann. of Math., 57 (1953), 287-308.

[9] _ Diffusion processes in one dimension, Trans. Amer. Math. Soc., 77 (1954), $1-31$.

[10] The general diffusion operator and positivity preserving semigroups in one dimension, Ann. of Math., 60 (1954), 417-436.

[11] K. Floret, Weakly Compact Sets, Lecture Notes in Mathematics No. 801, SpringerVerlag, 1980.

[12] E. Hille and R. S. Phillips, Functional Analysis and Semi-Groups, Amer. Math. Soc. Colloq. Publications, XXXI, New York, 1957.

[13] J. Hoffmann-Jørgensen, The Theory of Analytic Spaces, Various Publication Series No. 10, Aarhus Universitet, 1970.

[14] B. Jefferies, Bornology, Vector Measures and Cylindrical Measures, Ph.D. thesis, The Flinders University of South Australia, 1981.

[15] I. Kluvánek, Characterization of Fourier-Stieltjes transforms of vector and operatorvalued measures, Czechoslovak Math. J., 17 (92), (1967), 261-276.

[16] Yu. I. Lyubich, The classical and local Laplace transformation in an abstract Cauchy problem, Uspehi Mat. Nauk, 21 (1966), 3-51; English translation, Russian Math. Surveys, 21 (3), (1966), 1-52.

[17] I. Miyadera, On the representation theorem by the Laplace transformation of vectorvalued functions, Tôhoku Math. J., 8 (1956), 170-180.

[18] S. Okada, Integration of Vector-Valued Functions, Lecture Notes in Mathematics No. 1033, Measure theory and its applications, Proc. of Sherbrooke Conference, Springer-Verlag, 1982.

[19] P. G. Rooney, An inversion and representation theory for the Laplace integral of abstractly-valued functions, Canad. J. Math., 6 (1954), 190-209.

[20] L. Schwartz, Radon Measures on Arbitrary Topological Spaces and Cylindrical Measures, Oxford University Press, 1973.

[21] G. E. F. Thomas, Integration of functions with values in locally convex Suslin spaces, Trans. Amer. Math. Soc., 212 (1975), 61-81.

[22] H. von Weizsacker, Strong Measurability, Liftings and the Choquet-Edgar Theorem, Lecture Notes in Mathematics No. 645, Vector Space Measures and Applications II, Proc. of Dublin Conference, Springer-Verlag, 1977.

[23] A. K. Whitford, Characterization of Vector-Valued Laplace Transforms and Moment Sequences, Ph.D. thesis, The Flinders University of South Australia, 1972.

[24] D. V. Widder, The Laplace Transform, Princeton University Press, 1946.

[25] S. Zaidman, On the representation of vector-valued functions by Laplace transforms, Duke Math. J., 26 (1959), 189-191.

Received November 22, 1982 and in revised form January 31, 1984.

THE UNIVERSITY OF ADELAIDE

ADELAIDE

South Australia, 5000, Australia 


\title{
PACIFIC JOURNAL OF MATHEMATICS EDITORS
}

\author{
Donald BABBITt (Managing Editor) \\ University of California \\ Los Angeles, CA 90024 \\ J. DugunduI \\ University of Southern California \\ Los Angeles, CA 90089-1113 \\ R. FINN \\ Stanford University \\ Stanford, CA 94305 \\ HermanN FlaschKa \\ University of Arizona \\ Tucson, AŻ 85721
}

C. C. Moore

University of California

Berkeley, CA 94720

ARTHUR Ogus

University of California

Berkeley, CA 94720

Hugo Rossi

University of Utah

Salt Lake City, UT 84112

H. SAMELSON

Stanford University

Stanford, CA 94305

ASSOCIATE EDITORS
R. ARENS
E. F. BECKENBACH
B. H. NEUMANN
F. WOLF
K. YoSHIDA (1906-1982)

\section{SUPPORTING INSTITUTIONS}

$\begin{array}{ll}\text { UNIVERSITY OF ARIZONA } & \text { UNIVERSITY OF OREGON } \\ \text { UNIVERSITY OF BRITISH COLUMBIA } & \text { UNIVERSITY OF SOUTHERN CALIFORNIA } \\ \text { CALIFORNIA INSTITUTE OF TECHNOLOGY } & \text { STANFORD UNIVERSITY } \\ \text { UNIVERSITY OF CALIFORNIA } & \text { UNIVERSITY OF HAWAII } \\ \text { MONTANA STATE UNIVERSITY } & \text { UNIVERSITY OF TOKYO } \\ \text { UNIVERSITY OF NEVADA, RENO } & \text { UNIVERSITY OF UTAH } \\ \text { NEW MEXICO STATE UNIVERSITY } & \text { WASHINGTON STATE UNIVERSITY } \\ \text { OREGON STATE UNIVERSITY } & \text { UNIVERSITY OF WASHINGTON }\end{array}$

The Supporting Institutions listed above contribute to the cost of publication of this Journal, but they are not owners or publishers and have no responsibility for its content or policies.

Mathematical papers intended for publication in the Pacific Journal of Mathematics should be in typed form or offset-reproduced (not dittoed), double spaced with large margins. Please do not use built up fractions in the text of the manuscript. However, you may use them in the displayed equations. Underline Greek letters in red, German in green, and script in blue. The first paragraph must be capable of being used separately as a synopsis of the entire paper. In particular it should contain no bibliographic references. Please propose a heading for the odd numbered pages of less than 35 characters. Manuscripts, in triplicate, may be sent to any one of the editors. Please classify according to the scheme of Math. Reviews, Index to Vol. 39. Supply name and address of author to whom proofs should be sent. All other communications should be addressed to the managing editor, or Elaine Barth, University of California, Los Angeles, California 90024.

There are page-charges associated with articles appearing in the Pacific Journal of Mathematics. These charges are expected to be paid by the author's University, Government Agency or Company. If the author or authors do not have access to such Institutional support these charges are waived. Single authors will receive 50 free reprints; joint authors will receive a total of 100 free reprints. Additional copies may be obtained at cost in multiples of 50 .

The Pacific Journal of Mathematics is issued monthly as of January 1966. Regular subscription rate: $\$ 190.00$ a year (5 Vols., 10 issues). Special rate: $\$ 66.00$ a year to individual members of supporting institutions.

Subscriptions, orders for numbers issued in the last three calendar years, and changes of address should be sent to Pacific Journal of Mathematics, P.O. Box 969, Carmel Valley, CA 93924, U.S.A. Old back numbers obtainable from Kraus Periodicals Co., Route 100, Millwood, NY 10546.

The Pacific Journal of Mathematics at P.O. Box 969, Carmel Valley, CA 93924 (ISSN 0030-8730) publishes 5 volumes per year. Application to mail at Second-class postage rates is pending at Carmel Valley, California, and additional mailing offices. Postmaster: Send address changes to Pacific Journal of Mathematics, P.O. Box 969, Carmel Valley, CA 93924.

PUBLISHED BY PACIFIC JOURNAL OF MATHEMATICS, A NON-PROFIT CORPORATION

Copyright $@ 1984$ by Pacific Journal of Mathematics 


\section{Pacific Journal of Mathematics}

\section{Vol. 115, No. $2 \quad$ October, 1984}

Ersan Akyildiz, Gysin homomorphism and Schubert calculus ...........257

Marilyn Breen, Clear visibility and unions of two starshaped sets in the

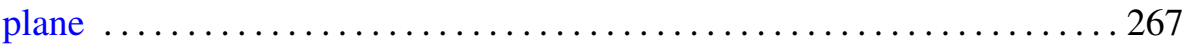

Robert F. Brown, Retraction methods in Nielsen fixed point theory . ......277

Herbert Busemann and Bhalchandra B. Phadke, A general version of Beltrami's theorem in the large ............................... 299

Gerald Arthur Edgar and Robert Francis Wheeler, Topological properties of Banach spaces ............................... 317

Yaakov Friedman and Bernard Russo, Conditional expectation without

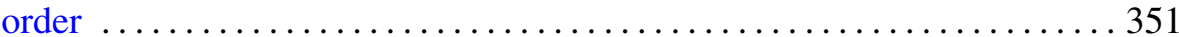

Robert Allen Goggins, Cobordism of manifolds with strong almost tangent

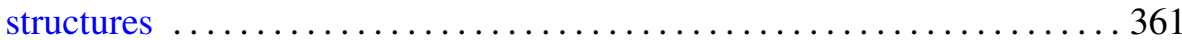

Mike Hoffman, Noncoincidence index of manifolds . . . . . . . . . . . . . 373

William H. Julian, $\varepsilon$-continuity and monotone operations $\ldots \ldots \ldots \ldots 385$

Gerasimos E. Ladas, Y. G. Sficas and I. P. Stavroulakis, Nonoscillatory

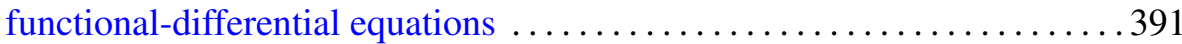

Arnold William Miller and Karel Libor Prikry, When the continuum has

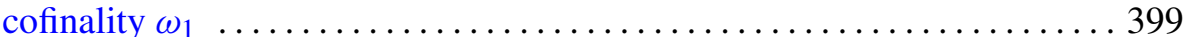

Jean-Leah Mohrherr, Density of a final segment of the truth-table

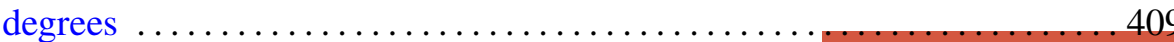

Carl Norman Mutchler, The flat Cauchy problem for radially hyperbolic operators from a characteristic manifold of high codimension ...

Kenji Nakagawa, On the orders of automorphisms of a closed Riemann surface

W. Ricker, Representation of vector-valued functions by Laplace transforms

Jorge Donato Samur, On semigroups of convolution operators in Hilbert

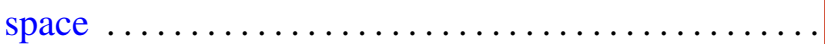

Joseph Gail Stampfli, One-dimensional perturbations of operators 481 Andrew George Earnest and John Sollion Hsia, Correction to: "Spinor norms of local integral rotations. II" 